\title{
Vaccines for COVID-19: Perspectives, Prospects, and Challenges Based on Candidate SARS, MERS, and Animal Coronavirus Vaccines
}

Authors:

Disclosure:

Acknowledgements:

Received:

Accepted:

Keywords:

Citation:
Linda J. Saif1,2,3,4

1. Food Animal Health Research Program, Ohio, USA

2. Ohio Agricultural Research \& Development Center (OARDC), Ohio, USA

3. College of Food, Agricultural, and Environmental Sciences (CFAES), Ohio, USA

4. Veterinary Preventive Medicine Department, College of Veterinary Medicine, The

Ohio State University, Wooster, Ohio, USA

The author has declared no conflicts of interest.

Salaries and research support were provided by state and federal funds appropriated to the Ohio Agricultural Research and Development Center, College of Food, Agricultural and Environmental Sciences, The Ohio State University, Wooster, Ohio, USA. Part of this work was supported by a grant from the US National Institutes of Health, NICHD Grant HD095881-01 (L. J. Saif and A. Vlasova, co-PIs).

03.03 .2020

13.03.2020

Animal coronavirus, coronavirus (CoV), COVID-19, Middle East respiratory syndrome (MERS), passive immunotherapies, severe acute respiratory syndrome (SARS), vaccines.

EMJ. 2020;DOI/10.33590/emj/200324

\section{INTRODUCTION}

Several coronaviruses (CoV) are widespread in humans and cause only mild upper respiratory infections and colds; however, pandemic outbreaks of more severe coronavirus infections in humans have become more prevalent. The severe acute respiratory syndrome (SARS) coronavirus (betaCoV Lineage B) caused the first pandemic of the $21^{\text {st }}$ century in 2002-2003, with its epicentre in China. The Middle East respiratory syndrome (MERS) coronavirus (betaCoV Lineage C) emerged almost a decade later and infections continue in the Middle East. Now, only 7 years after MERS, the COVID-19 SARS-CoV-2 (betaCoV Lineage B) has emerged, again in China, as an even more devastating pandemic. Its occurrence was not unexpected, because like SARS, for which the host origin was bats, scientists had previously identified SARS-like CoV in these animals in China.' Based on sequence analysis of the SARS-CoV-2 genome, it is more closely related to SARS (80\%) and to one bat RaTG13 SARS-like CoV (96\%) than to MERS CoV (54\%). ${ }^{2}$

To date we have a limited arsenal to combat these deadly infections, with no approved treatments or vaccines for any of these severe CoV diseases, including COVID-19. Research on these diseases was largely curtailed by lack of interest in vaccines among pharmaceutical companies and lack of sustained government funding, as SARS disappeared and MERS waned. COVID-19 and SARS-CoV bind the same host cell receptor (angiotensin converting enzyme 2 [ACE2]) and may share similar disease pathogeneses and limited cross-neutralising antibodies. ${ }^{2}$ This knowledge, as well as our improved understanding of CoV replication strategies and technological advancements in vaccines since SARS, ${ }^{3}$ suggests that a first-generation COVID-19 vaccine could be 
forthcoming more rapidly than before but unfortunately may not be available to stem the current outbreak.

SARS-CoV-2 has a large single stranded positive RNA genome (29.88Kb) with approximately 12 open reading frames. Like all CoV, it encodes genes for four major structural proteins, specifically the nucleocapsid (N) associated with the RNA genome and three membrane proteins: the large spike (S) glycoprotein (uncleaved in SARS, cleaved in SARS-CoV-2 and infectious bronchitis virus [IBV] into amino-terminal S1 and carboxy-terminal S2); the integral membrane (M) glycoprotein; and the envelope (E) protein. The $S$ protein contains the receptor binding domain (RBD) and functions in viral attachment (via S1) and membrane fusion (via S2) to host cells, as well as induction of neutralising ( $V N$ ) antibodies that can block binding to the host receptor. ${ }^{2}$ It is a major focus in vaccine development. For SARS-CoV, the $M$ protein can also induce $\mathrm{VN}$ antibodies and the $\mathrm{N}$ protein contains T-cell epitopes, meaning they may also be vaccine targets. $3,4,5$

\section{COVID-19, SARS, AND MERS CANDIDATE VACCINES}

No coronavirus vaccines to prevent respiratory infections in humans have been licensed. In animals, only IBV vaccines are licensed to prevent upper respiratory CoV infections in chickens. ${ }^{6,7}$ Like for SARS and MERS, several types of COVID-19 vaccines are proposed or in various stages of development. ${ }^{8}$ Major criteria for all vaccines include safety and efficacy plus duration of immunity, but vaccines for pandemics also necessitate rapid development and high production capacity. ${ }^{3}$ Although classical inactive and attenuated vaccines are being evaluated, rapid production of these virus vaccines in large quantities in cell culture under biosafety level-3 conditions is challenging, and this limits their rapid deployment in the face of emerging pandemics. However, live attenuated CoV vaccines generated by reverse genetics from infectious virus clones by deletion of multiple key virulence determinants to prevent reversion (ExoN, nsp16, accessory proteins, etc.) remain the most immunologically robust, inducing mucosal, systemic, humoral, and cell mediated immunity and broader crossprotection. ${ }^{4,5,6,9}$ As such, they are highly effective for priming immune responses in naïve hosts. ${ }^{6,10}$ An ideal approach for an attenuated COVID-19 vaccine would be to first generate a temperaturesensitive mutant virus with restricted replication to the upper respiratory tract, and then apply reverse genetics to construct additional targeted attenuating mutations. In combination with a parenteral heterologous $\mathrm{S}$ and $\mathrm{N}$ protein booster vaccine, such an attenuated vaccine could also potentially elicit crossprotection against heterologous strains that spill over within a betaCoV lineage, such as bat SARS-like strains. ${ }^{1}$

Newer vaccine technologies include viral proteins (subunits or virus-like particles), recombinant viral vectors, or nucleic acid vaccines. The latter two have the advantage of providing universal vaccine platforms amenable to introduction of new antigenic targets from emerging viruses. ${ }^{3}$ They mimic attenuated vaccines by infecting host cells or inducing endogenously produced antigenic proteins to generate both antibody and T-cell immune responses. ${ }^{3,4,5,9}$ COVID-19 candidate vaccines under development include $S$ protein or RBD subunit vaccines and replicating or non-replicating vector vaccines expressing mainly S protein or the RBD. ${ }^{8}$ Vaccines should be based on a consensus $S$ gene to account for variability among strains. Other vaccines are based on nucleic acid constructs such as DNA plasmids or mRNA vaccines that encode $\mathrm{S}$ or RBD proteins expressed in host cells. ${ }^{4,5,9}$ Although DNA vector vaccines may be safe, stable, and rapidly produced, their immunogenicity and efficacy in humans is not yet proven. SARS and MERS CoV DNA vaccines often have greater efficacy in DNA prime/ heterologous boost (S/S1 proteins, inactivated virus, or recombinant viral vectors) regimens. ${ }^{4,5,9}$ DNA vaccine administration by electroporation and its possible genomic integration and persistence are remaining issues. ${ }^{3}$ mRNA vaccines are used as templates for endogenous protein production in the vaccine recipient. Delivery of the mRNA vaccine is enhanced by use of lipid nanoparticles for intramuscular (IM) or intradermal administration. ${ }^{3}$ An mRNA vaccine for COVID-19 is the first to advance to initial Phase I safety trials in humans in the USA. A potential advantage of mRNA vaccines is the 
anticipated development of a portable mRNA 'printing' facility to produce large quantities of mRNA.

Recombinant vector vaccines in various stages of development for SARS or MERS include recombinant adenovirus (Ad) vectors with CHAd63 from chimpanzees used to overcome the widespread pre-existing immunity to human adenoviruses (Ad 5 etc.)., ${ }^{4,5}$ Recombinant Ad vectors expressing SARS-CoV $\mathrm{S}$ or $\mathrm{N}$ proteins or MERS-CoV S proteins elicited variable levels of protection in mouse, ferret, or nonhuman primate (NHP) challenge models. ${ }^{4,5,9}$ Other candidate vectored vaccines for SARS or MERS include poxvirus vectors (such as modified vaccinia ankara [MVA]), parainfluenza, measles virus, Newcastle disease virus (NCD), and vesicular stomatitis virus which express the SARS- or MERS-CoV $\mathrm{S}$ protein or $\mathrm{S}$ and $\mathrm{N}$ proteins. $4,5,9$ They induced variable levels of protection, as mostly accessed in mouse models, including transgenic mice expressing human ACE2 or dipeptidyl peptidase 4, the MERS-CoV host receptor. Notably some of the candidate vaccines (MVA and NCD MERS-CoV S) were also tested in the MERS intermediate animal host, dromedary camels, and shown to induce VN antibodies and, for MVA, protection against nasal shedding. ${ }^{9}$ It is important to design vaccines for livestock that serve as intermediate hosts to curtail spill over into humans. A similar vaccine strategy would entail use of poultry or swine influenza vaccines to limit transmission of potentially high-risk zoonotic influenza viruses to humans.

Safety is of major concern for vaccines and as such it is important to investigate adverse events or vaccine-induced immunopathology evident during candidate vaccine studies in animal models. Eosinophil-related lung pathology was observed in mice vaccinated with formalin and ultraviolet-inactivated SARS vaccine ${ }^{4}$ or $\mathrm{Y}$-irradiated inactivated MERS-CoV vaccine post-murine challenge; however, adding toll-like receptor agonists to an ultraviolet-inactivated SARS-CoV vaccine reduced the Th2-associated lung pathology. ${ }^{9}$ In one ferret study, the MVA-S vaccine was associated with liver pathology, but this was not evident in other studies. ${ }^{4}$ In tests of a SARS-S protein candidate vaccine, antibodydependent enhancement (ADE) of infection was reported post-challenge in hamsters, ${ }^{4}$ but not in mice using an $S$ protein nanoparticle vaccine for MERS. ${ }^{9}$ ADE has remained a long-term obstacle to the development of safe vaccines for feline infectious peritonitis, a systemic CoV infection of cats. ${ }^{6,10}$ In feline infectious peritonitis-infected cats, ADE was triggered by antibody-mediated virus entry into macrophages via Ig Fc receptors. The inconsistencies in these events among animal models necessitates an improved understanding of the biological basis for their occurrence and a better knowledge of human immunology to avoid similar reactions in humans.

\section{CORONAVIRUS VACCINE STRATEGIES BASED ON CORONAVIRUS PATHOGENESIS IN THE HOST}

COVID-19 vaccination strategies would be aided by a clearer understanding of SARSCoV-2 pathogenesis in humans, the correlates of protection, and the duration of natural immunity. An understanding of the pathogenesis of SARSCoV-2, including the target organs infected and the route of virus dissemination to these organs, will assist in development of vaccines to block viral dissemination and prevent infection of the target organs. An important consideration is if SARS-CoV-2 targets the lungs to cause pneumonia via viraemia or after an upper respiratory infection. If the latter, then IN vaccines using live replicating vectors or attenuated viruses that effectively induce local mucosal immunity could protect the upper and, consequently, the lower respiratory tracts and reduce nasal shedding. An example is the current use of a live attenuated influenza virus vaccine that induces mainly local IgA antibodies and fewer systemic antibodies, yet elicits protection."

Alternatively, if the lungs (or other organs) are the major sites of infection via viraemia, then parenteral (IM) vaccines that elicit sufficient VN antibodies in serum to block viraemia and are also transudated to the lungs (and other target organs) may effectively block infection. This would be equivalent to the IM application of inactivated influenza vaccine to prevent respiratory infections in humans. Additionally, in people who have recovered from COVID-19 and are primed to the virus (again, like seasonal influenza), a parental vaccine alone, such as 
a subunit $\mathrm{S}$ or RBD protein, may be effective as an annual booster vaccine. This would enhance memory $\mathrm{B}$ - and $\mathrm{T}$-cell responses and immunity, and prevent virus reinfections. Diarrhoea and foecal shedding were reported in some COVID-19 patients, ${ }^{12}$ so for this scenario, oronasal vaccines may be more effective. Thus, COVID-19 vaccines will likely be used in three populations: naïve susceptible individuals with no immunity; recovered, including subclinically infected, individuals, with various levels of immunity; and in people who have pre-existing immunity to SARS and MERS. Therefore the immunogenicity, protective potential, or adverse effects of candidate vaccines may vary among such populations. Assessing pre-existing levels of immunity will be important to validate vaccine effectiveness and safety in each population and in the various age groups within each population, but especially in the elderly with the highest death rates.

\section{ANIMAL MODELS FOR SARS, MERS, AND COVID-19 CORONAVIRUSES}

A variety of small animal models have been used to test vaccines for SARS- and MERSCoV. 4,5,9 They include NHP (macques, African Green monkeys), mice, hamsters, and ferrets. These as well as additional animals, including susceptible livestock species, should be studied to define which models best mimic human COVID-19 infections and the potential correlates of protection. As with most animal models, none fully recapitulate the disease pathogenesis in humans or replicate human physiology and immune responses: all have advantages and limitations. The robustness of inbred mice models that do not reproduce SARS or MERS disease has been improved by the use of aged mice, mouse-adapted SARS or MERS strains, and hACE2 or DPP4 transgenic mice, respectively. ${ }^{4,5}, 9$ Ferrets, also used as an influenza model, reflect SARS pathogenesis in humans including fever, nasal shedding, and lung pathology; ${ }^{4}$ however, their less wellcharacterised immune systems and lack of reagents compared to mouse models are a limitation. Pigs are susceptible to infection with MERS-CoV ${ }^{13}$ and SARS ${ }^{14}$ and and if susceptible to SARS-CoV-2, they are potentially a relevant model because they are outbred and their physiology, metabolism, respiratory anatomy, and immune responses resemble those of humans. ${ }^{15}$ Although NHP better reflect humans, they too do not manifest all of the clinical signs, disease, and immune parameters in humans and are limited in availability.

With all of these models, the major concern is how well they will predict vaccine immune responses, including adverse events, and protection in humans. Because clinical trials are now underway for three MERS vaccines, ${ }^{8}$ once they are tested in humans in the ongoing MERS outbreak settings, the results should reveal how well data from these models predict human responses to CoV and protection. Much could be learned about CoV pathogenesis, factors that influence shedding and transmission, and safety and immunity induced by candidate vaccines in the susceptible intermediate host species. Although currently unknown for COVID-19, only limited studies have been done in camels infected or vaccinated with MERSCoV. ${ }^{9}$ Use of an effective MERS vaccine in camels is also an important strategy to block ongoing transmission to humans.

\section{LESSONS FROM ANIMAL CORONAVIRUS VACCINES}

To prevent CoV infections in livestock and poultry, most of the current licensed CoV vaccines are either inactive, attenuated, or live vector (porcine epidemic diarrhoea virus) vaccines (comparisons with SARS and testing of new-generation vaccines are reviewed ${ }^{6,10}$ ). None are completely efficacious in animals. The gastrointestinal tract is the major site of CoV infection in many animals, and severity is greatest in neonates. As such, oral attenuated vaccines were developed for use in pregnant animals, both to prevent disease in the mother and also to induce high levels of passive IgA antibodies in milk that are transferred to neonates via suckling to prevent intestinal infection. In studies of swine, milk IgA, but not serum IgG antibodies were correlates of passive immunity to enteric CoV infections of neonates. ${ }^{6,10}$

The only licensed animal CoV vaccines targeted to prevent respiratory CoV infections are IBV vaccines for chickens. ${ }^{6,7,0}$ However, unlike 
SARS-CoV-2 which causes atypical pneumonia, IBV causes an upper respiratory infection with infection of bronchi, severe disease in young chicks, and infection of the kidney and reproductive tract by some strains. Both live attenuated and inactivated IBV vaccines are licensed, with the latter also used in an attenuated prime/inactivated boost vaccine regimen. The correlates of protection against IBV clinical disease are uncertain, but high levels of serum VN antibodies are suggested to prevent viral dissemination from the respiratory tract, thus blocking infection of the reproductive tract and kidneys. Generally, live attenuated or certain replicating vectored vaccines were more effective in fewer doses than inactivated IBV or subunit vaccines. Problems encountered in vaccine protection include the existence of multiple serotypes/ subtypes of IBV which fail to cross-protect, variation in virulence among IBV field strains, and reduced but not eliminated nasal shedding.

Bovine CoV (BCoV) is pneumoenteric and causes diarrhoea and respiratory disease in cattle..$^{6,10}$ Upper respiratory reinfections are common with repeated nasal shedding episodes. It is endemic and most cattle are seropositive, but antibody titers wane unless boosted by reinfections or vaccines. In spite of its economic impact, no respiratory vaccines have been developed to prevent BCoV-associated pneumonia in calves or in feedlot cattle. In cattle naturally infected with BCoV, high serum antibody titers have been correlated with protection of feedlot cattle against BCoV-induced pneumonia and shedding associated with the bovine respiratory disease complex. Also application of a live attenuated $\mathrm{BCoV}$ vaccine (licensed for oral use to prevent $\mathrm{BCoV}$ diarrhoea) in cattle on entry to a feedlot reduced the risk of treatment for bovine respiratory disease complex. Because MERS-CoV is endemic in camels (which, like cattle, are ruminants) in Saudi Arabia and camels are mostly seropositive to MERS-CoV, a similar approach, but using an IM or IN S protein subunit or inactivated MERS-CoV as a safe booster vaccine, could be successful to reduce virus shedding and transmission to humans.

The porcine respiratory coronavirus (PRCV) resembles SARS-CoV-2 infections in many important clinicopathological aspects (aerogenic spread via droplets, tropism for the lung, and interstitial pneumonia affecting $5-60 \%$ of the lung). ${ }^{6,10}$ Despite the lung lesions, many PRCV infections are clinically mild. Respiratory coinfections, dose, route of infection, and immunosuppression (corticosteroids) are cofactors that exacerbate the severity of PRCV and also BCoV infections.6,10 These cofactors may play a role in the severity of COVID-19 or enhanced virus transmission by superspreaders. Although no vaccines have been developed for PRCV, an Ad5 vector vaccine expressing the PRCV S protein inoculated oronasally into pigs reduced but did not prevent PRCV nasal shedding and elicited a rapid anamnestic $\mathrm{VN}$ antibody response post-challenge.

\section{CONCLUSIONS AND CHALLENGES FOR COVID-19 VACCINEDEVELOPMENT}

In the face of a pandemic, rapid development, production, and deployment of first-generation vaccines are critical. Synthetic nucleic acid (DNA, mRNA) priming vaccines in combination with $S$ (and possibly $N$ ) protein booster vaccines are leading candidates based on the above criteria. An approach used to expedite veterinary vaccines during epidemics is to issue conditional licensures; for COVID-19 these could be based on human clinical data confirming safety and adequate levels of protection to reduce fatalities in the highest risk groups (elderly and patients with comorbidities, healthcare workers). Second-generation, more potent, or efficacious vaccines to prevent disease, deaths, and reduce shedding, as discussed, should be developed in parallel for future deployment.

Most candidate vaccines are predicated on the induction of serum VN antibodies and systemic cell-mediated immune responses in the animal models as indicators of protection, but the correlates of immunity to COVID-19 in humans are unknown. Mucosal immune responses may be important, particularly to reduce nasal shedding. A possible scenario is that vaccines will prevent severe disease and deaths, but may not eliminate nasal shedding, allowing continued transmission. Achieving sterilising immunity at mucosal surfaces is a major challenge to prevent virus shedding and mucosal immunity is often short-lived, requiring multiple booster vaccine doses. 
The elderly and those with chronic conditions or comorbidities are at greater risk of severe disease or mortality, yet many existing vaccines (such as for influenza) have reduced efficacy in these groups. Alternative vaccination approaches such as better adjuvants and multiple or higher doses, like for high-dose inactivated influenza vaccines, may be needed to confer protection in these vulnerable groups. Animal models also need to mimic these parameters.

The pathogenesis of COVID-19 in humans is unclear, and as such vaccine strategies may need to be altered if the virus infects both the respiratory and intestinal tracts (pneumoenteric, like BCoV) and is also shed in faeces. Oronasal vaccine prime and parenteral $\mathrm{S}$ vaccine booster may be optimal to prevent both enteric and respiratory infections and faecal and nasal shedding as used for some animal CoV vaccines.

Future spill over of SARS or SARS-CoV-2like CoVs from animal reservoirs is likely. New approaches are needed to generate vaccines that can induce broader heterologous and cross-protective immunity against $\mathrm{CoV}$ within each betaCoV lineage. This will require focus on additional proteins (S2, N, etc.) and conserved epitopes that induce broad cross-reactive and cross-protective immunity.
The lack of vaccines to induce active immunity warrants the rapid development and application of passive immunisation approaches to treat patients both prophylactically and therapeutically. The most timely would be convalescent plasma therapy using plasma containing antibodies from recovered patients as an empirical treatment during the COVID-19 outbreak followed by confirmatory double blind trials. A shorter hospital stay and reduced mortality was reported in plasma-treated versus untreated SARS patients. ${ }^{16}$ If effective, plasma banks with blood donated by the substantial numbers of recovered COVID-19 individuals could be established promptly.

Monoclonal antibodies (MAB) against the target viral proteins can be produced rapidly and are important to map the epitopes that confer protective and cross-reactive immune responses as confirmed by administration of the MAB to SARS-CoV-2 challenged animals. The protective MAB can guide vaccine design and most importantly can provide passive immunoprophylactics for COVID-19 in patients. ${ }^{17}$ A recent relevant strategy used mRNA encoding respiratory syncytial virus MAB (palinozumab) delivered to the lung via intratracheal aerosols. ${ }^{18}$ Nanobodies (camelid variable heavy-chain antibodies) developed for MERS provided passive immunotherapy in a mouse model, and represent another promising approach. ${ }^{19}$

\section{References}

1. Ng OW, Tan YJ. Understanding bat SARS-like coronaviruses for the preparation of future coronavirus outbreaks - Implications for coronavirus vaccine development. Hum Vaccine Immunother. 2017;13(1):186-9.

2. Zhou $P$ et al. A pneumonia outbreak associated with a new coronavirus of probable bat origin. Nature. 2020;579:270-3.

3. Rauch $\mathrm{S}$ et al. New vaccine technologies to combat outbreak situations. Front Immunol. 2018;9:1963.

4. Roper RL, Rehm KE. SARS vaccines: where are we? Expert Rev Vaccines. 2009;8(7):887-98

5. Enjuanes L et al. Vaccines to prevent severe acute respiratory syndrome coronavirus-induced disease. Virus Res. 2008;133(1):45-62.
6. Saif LJ. "Coronaviruses of domestic livestock and poultry: Interspecies transmission, pathogenesis and immunity", Perlman, S et al (eds), Nidovirales (2017), Washington, DC: American Society of Microbiology, pp. 279-98.

7. Jordan B. Vaccination against infectious bronchitis virus: a continuous challenge. Vet Microbiol 2017;206:137-43.

8. World Health Organization (WHO). Coronavirus disease (COVID-2019) R\&D. Available at: https://www.who. int/blueprint/priority-diseases/keyaction/novel-coronavirus/en/. Last Accessed: 13 March 2020.

9. Schindewolf C, Menachery VD. Middle east respiratory syndrome vaccine candidates: cautious optimism. Viruses. 2019;11(1). pii: E74.

10. Saif LJ, "Animal coronaviruses: lessons for SARS”. Knobler S et al. (eds) Learning from SARS: Preparing for the next disease outbreak (2004). Washington, DC: National Academies Press, pp. 138-49..

11. Rudraraju $\mathrm{R}$ et al. How live attenuated vaccines can inform the development of broadly cross-protective influenza vaccines. J Infect Dis. 2019;219 (Supplement1):S81-7.

12. Wölfel R et al. Virological assessment of hospitalized cases of coronavirus disease 2019. medRxiv preprint doi:https://doi.org/10.1101/2020.03.05 .20030502

13. Vergara-Alert $\mathrm{J}$ et al. Livestock susceptibility to infection with middle east respiratory syndrome coronavirus. Emerg Infect Dis. 2017;23(2):232-40.

14. Chen $W$ et al. 2005. SARS-associated coronavirus transmitted from human 
to pig. Emerg Infect Dis 2005:11:446448

15. Vlasova A N et al, "Gnotobiotic neonatal pig model of rotavirus infection and disease," Svensson L et al. (eds), Viral gastroenteritis: molecular epidemiology and pathogenesis (2016). The Netherlands: Elsevier, pp. 219-41.
16. Cheng $Y$ et al. Use of convalescent plasma therapy in SARS patients in Hong Kong. Eur J Clin Microbiol Infect Dis. 2005;24(1):44-6.

17. Wang $\mathrm{C}$, et al $\mathrm{A}$ human monoclonal antibody blocking SARS-CoV-2 infection. 2020. bioRxiv preprint doi: https://doi. org/10.1101/2020.03.11.987958
18. Tiwari PM et al. Engineered mRNAexpressed antibodies prevent respiratory syncytial virus infection. Nat Commun. 2018;9(1):3999.

19. Stalin R V et al. Chimeric camel/ human heavy-chain antibodies protect against MERS-CoV infection. Sci Adv. 2018;4(8):eaas9667. 